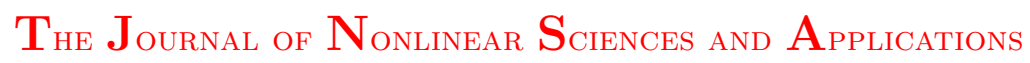

http://www.tjnsa.com

\title{
IMPULSIVE STABILIZATION OF CELLULAR NEURAL NETWORKS WITH TIME DELAY VIA LYAPUNOV FUNCTIONALS
}

\author{
QING WANG ${ }^{1}$ AND XINZHI LIU ${ }^{2 *}$
}

\begin{abstract}
This paper investigates the problem of global exponential stability for a class of impulsive cellular neural networks with time delay. By employing Lyapunov functionals, some sufficient conditions for exponential stability are established. Our results show that unstable cellular neural networks with time delay may be stabilized by impulses, where the upper bound of the amplitudes of the impulses is given. Numerical simulations on two examples are given to illustrate our results.
\end{abstract}

\section{INTRODUCTION}

Cellular neural networks, introduced by Chua and Yang [8], have been extensively investigated recently due to their important applications in such fields as image processing, pattern recognition and quadratic optimization. Time delays seem inevitable in the signal transmission among neurons and may affect the performance of the neural system. As a consequence, delayed cellular neural networks have attracted the attention of many scientists and there has been a considerable number of research work on the cellular neural networks with time

Date: Received: 5 August 2008; Revised: 15 August 2008.

* Corresponding author.

2000 Mathematics Subject Classification. Primary 34d20; Secondary 44B20, 46C05.

Key words and phrases. Impulsive cellular neural networks, global exponential stability, stabilization, time delay. 
delay, see [2, 4, 9, 11] for example. Those delayed cellular neural networks models have found potential applications in various types of motion-related processes such as processing of moving images, pattern classification, moving objects speed detection, quadratic optimization, robotics and control, see [5, 6, 14, 15]. Besides delay effect, many evolutionary processes in areas such as biology, electronics, mechanics, economics and telecommunications undergo abrupt state changes at certain moments of time due to impulsive inputs. Such systems are described by impulsive differential equations, which have been successfully introduced to the modelling of neural networks $([1,10,19,21])$.

Many stability criteria for delayed cellular neural networks without impulses have been obtained in [2, 9, 11, 20], [4]-[7], [13]-[17], where many methods such as Lyapunov method, linear matrix inequality (LMI), $M$-matrix, Razumkhin technique and differential inequalities have been used. In the work mentioned above, the global exponential stability of the equilibrium point is one of the most investigated problems of cellular neural networks. If an equilibrium of a cellular neural network is globally exponentially stable, it means that the domain of attraction of the equilibrium point is the whole space which in turn implies that this equilibrium point is unique and all other solutions converge to it very quickly. This is of importance from both theoretical and practical point of view. Such cellular neural networks are known to be well-suited for solving some optimization problems. For example, a globally exponentially stable cellular neural network is guaranteed to compute the global optimal solution independently of the initial condition and hence is devoid of spurious suboptimal response.

On the other hand, there is much less work done for stability investigation of impulsive cellular neural networks with time delay. Recently many interesting results have been obtained for dealing with the effect of impulses on the stability properties of the delayed Cellular neural networks. In earlier works, in order to keep the good stability properties of the neural networks without impulses, the amplitudes of impulses are assumed to be bounded by those of solutions right before the impulse moments (see [1] for example), then in [19], the assumption on the amplitudes of impulses are relaxed a bit to be bound by those of some series with bounds not less than those of the solutions right before the impulse moments, and in [21], the amplitudes of impulses can even be unbounded. Nevertheless, there has been very few work devoted to the impulsive stabilization of the cellular neural networks with time delay. The objective of this paper is to develop some results in this regard. We shall use the method of Lyapunov functionals to establish exponential stability criteria for a class of impulsive delayed cellular neural networks utilizing the ideas developed in [12, 18, 19]. Our results 
show that impulses may be used to stabilize the cellular neural networks with time delay if they are not stable. Two examples with computer simulations are also given to illustrate our results.

\section{Preliminaries}

Let $\mathbb{R}$ denote the set of real numbers, $\mathbb{R}_{+}$the set of nonnegative real numbers and $\mathbb{R}^{n}$ the $n$-dimensional real space equipped with the norm $\|x\|=\left(\sum_{i=1}^{n}\left|x_{i}\right|^{p}\right)^{\frac{1}{p}}$ with $x=\left(x_{1}, x_{2}, \cdots, x_{n}\right)^{T}$ and $p=1$ or 2 . Let $\mathbb{N}^{*}$ denote the set of positive integers, i.e., $\mathbb{N}^{*}=\{1,2, \cdots\}$. Denote $\psi\left(t^{+}\right)=\lim _{s \rightarrow t^{+}} \psi(s)$ and $\psi\left(t^{-}\right)=$ $\lim _{s \rightarrow t^{-}} \psi(s)$. For $a, b \in \mathbb{R}$ with $a<b$ and for $S \subset \mathbb{R}^{n}$, we define

$$
\begin{aligned}
P C([a, b], S)= & \left\{\psi:[a, b] \rightarrow S \mid \psi(t)=\psi\left(t^{+}\right), \forall t \in[a, b) ; \psi\left(t^{-}\right) \text {exists in } S, \forall t\right. \\
& \in(a, b], \text { and } \psi\left(t^{-}\right)=\psi(t) \text { for all but at most a finite number } \\
& \text { of points } t \in(a, b]\} .
\end{aligned}
$$

Given a constant $\tau>0$, we equip the linear space $P C\left([-\tau, 0], \mathbb{R}^{n}\right)$ with the norm $\|\cdot\|_{\tau}$ defined by $\|\psi\|_{\tau}=\sup _{-\tau \leq s \leq 0}\|\psi(s)\|$.

Consider the impulsive delayed cellular neural networks described by the following impulsive delay differential equations

$$
\left\{\begin{aligned}
\frac{d u_{i}(t)}{d t}= & -c_{i} u_{i}(t)+\sum_{j=1}^{n} a_{i j} f_{j}\left(u_{j}(t)\right)+\sum_{j=1}^{n} b_{i j} f_{j}\left(u_{j}\left(t-\tau_{j}\right)\right) \\
& +J_{i}, \quad t \in\left[t_{k-1}, t_{k}\right), \\
\Delta u_{i}\left(t_{k}\right)= & I_{i k}\left(u_{i}\left(t_{k}^{-}\right)\right), \quad k \in \mathbb{N}^{*} \\
u_{i t_{0}} & =\psi_{i}, \quad i=1,2, \cdots, n .
\end{aligned}\right.
$$

where $u_{i}(\cdot)$ is the state representing the membrane potential of the $i^{\text {th }}$ unit; $J_{i}$ is a constant denoting the external bias or input from outside the network to the $i^{\text {th }}$ unit; $a_{i j}, b_{i j}$ are constants; where $\tau_{i}$, bounded by $\tau$, are constants denoting the transmission delay; $n$ corresponds to the number of units in a neural network; $f_{i}: P C([-\tau, 0], \mathbb{R}) \rightarrow \mathbb{R}$ is the activation function satisfying

$$
\begin{gathered}
\left|f_{i}\left(u_{i}\right)\right| \leq N_{i}, \quad \forall u_{i} \in \mathbb{R}, \\
0 \leq \frac{f_{i}\left(u_{i}\right)-f_{i}\left(v_{i}\right)}{u_{i}-v_{i}} \leq L_{i}, \quad \forall u_{i} \neq v_{i}, u_{i}, v_{i} \in \mathbb{R}, i=1,2, \cdots, n .
\end{gathered}
$$

And $\phi_{i} \in P C([-\tau, 0], \mathbb{R})$ is the initial function; $I_{i k} \in P C([-\tau, 0], \mathbb{R})$ represents the effects of impulsive control or perturbation; $t_{k}$ is impulsive moment and $0 \leq$ $t_{0}<t_{1}<t_{2}<\cdots<t_{k}<\cdots$, with $t_{k} \rightarrow \infty$ as $k \rightarrow \infty ; \Delta u_{i}(t)=u_{i}\left(t^{+}\right)-u_{i}\left(t^{-}\right)$; and $u_{i t}, u_{i t^{-}} \in P C([-\tau, 0], \mathbb{R})$ are defined by $u_{i t}(s)=u_{i}(t+s), u_{i t^{-}}(s)=u_{i}\left(t^{-}+s\right)$ for $-\tau \leq s \leq 0$, respectively. 
From [3], we know that the system (2.1) without impulses(or $I_{i k}(s)=s$ for any $s \in \mathbb{R})$ has at least one equilibrium point if conditions (2.2) and (2.3) hold. Denote one of the equilibrium points by $u^{*}=\left[u_{1}^{*}, u_{2}^{*}, \cdots, u_{n}^{*}\right]^{T}$, we shall investigate the global exponential stability of this equilibrium point $u^{*}$. The concept of global exponential stability is defined as follows.

Definition 2.1. An equilibrium $u^{*}$ of system (2.1) is said to be globally exponentially stable, if for any initial data $u_{t_{0}}=\phi \in P C\left([-\tau, 0], \mathbb{R}^{n}\right)$, there exist some $M, \alpha>0$ such that

$$
\left\|u\left(t, t_{0}, \phi\right)-u^{*}\right\| \leq M\left\|\phi-u^{*}\right\|_{\tau} e^{-\alpha\left(t-t_{0}\right)}, \quad \text { for all } t \geq t_{0},
$$

where $t_{0} \in \mathbb{R}_{+}$.

Define $x_{i}(\cdot)=u_{i}(\cdot)-u_{i}^{*}$ and then system (2.1) can be simplified as

$$
\left\{\begin{array}{lll}
\frac{d x_{i}(t)}{d t} & =-c_{i} x_{i}(t)+\sum_{j=1}^{n} a_{i j} f_{j}\left(x_{j}(t)\right)+\sum_{j=1}^{n} b_{i j} f_{j}\left(x_{j}\left(t-\tau_{j}\right)\right), & t \geq t_{0}, t \neq t_{k}, \\
\Delta x_{i}\left(t_{k}\right) & =I_{i k}\left(x_{i}\left(t_{k}^{-}\right)+u_{i}^{*}\right), \quad k \in \mathbb{N}^{*} & \\
x_{i t_{0}} & =\phi_{i}-u_{i}^{*}, \quad i=1,2, \cdots, n,
\end{array}\right.
$$

where $f_{j}\left(x_{j}(\cdot)\right)=f_{j}\left(x_{j}(\cdot)+u_{j}^{*}\right)-f_{j}\left(u_{j}^{*}\right)$. Assume $I_{i k}\left(u_{i}^{*}\right)=0$ so that system (2.5) admits the trivial solution. So the stability problem of the equilibrium point $u^{*}$ of system (2.1) is equivalent to the stability problem of the trivial solution of system (2.5).

\section{MAIN RESUlts}

In this section, we shall discuss the global exponential stability for the equilibrium point $u^{*}$ of system (2.1).

Theorem 3.1. Assume that there exist constants $l, \alpha, d>0$ such that $\tau \leq$ $t_{k}-t_{k-1} \leq l,\left|I_{i}\left(y+u_{i}^{*}\right)+y\right| \leq d|y|$ for any $y \in \mathbb{R}$ and $i=1,2, \cdots, n$ with

$$
\ln (d+\lambda \tau) \leq-(\alpha+c) l,
$$

where $c=\max _{1 \leq i \leq n}\left\{-c_{i}+\sum_{j=1}^{n}\left(\left|a_{j i}\right|+\left|b_{j i}\right|\right) L_{i}\right\}>0$, and $\lambda=\max _{1 \leq j \leq n}\left\{\sum_{i=1}^{n}\right.$ $\left.\left|b_{i j}\right| L_{j}\right\}$, then the equilibrium point $u=u^{*}$ of system (2.1) is globally exponentially stable.

Proof. Choose Lyapunov functional

$$
V\left(t, x_{t}\right)=\sum_{i=1}^{n}\left(\left|x_{i}(t)\right|+\sum_{j=1}^{n}\left|b_{i j}\right| L_{j} \int_{t-\tau_{j}}^{t}\left|x_{j}(s)\right| d s\right),
$$


then the Dini derivative of $V$ along system (2.5) is

$$
\begin{aligned}
\frac{d V\left(t, x_{t}\right)}{d t} \leq & \sum_{i=1}^{n}\left[-c_{i}\left|x_{i}(t)\right|+\sum_{j=1}^{n}\left|a_{i j}\right| L_{j}\left|x_{j}(t)\right|\right. \\
& \left.+\sum_{j=1}^{n}\left|b_{i j}\right| L_{j}\left|x_{j}\left(t-\tau_{j}\right)\right|+\sum_{j=1}^{n}\left|b_{i j}\right| L_{j}\left(\left|x_{j}(t)\right|-\left|x_{j}\left(t-\tau_{j}\right)\right|\right)\right] \\
= & \sum_{i=1}^{n}\left[-c_{i}+\sum_{j=1}^{n}\left(\left|a_{j i}\right|+\left|b_{j i}\right|\right) L_{i}\right]\left|x_{i}(t)\right| \\
\leq & c V\left(t, x_{t}\right), \quad t \in\left[t_{k-1}, t_{k}\right), k \in \mathbb{N}^{*},
\end{aligned}
$$

where $c=\max _{1 \leq i \leq n}\left\{-c_{i}+\sum_{j=1}^{n}\left(\left|a_{j i}\right|+\left|b_{j i}\right|\right) L_{i}\right\}>0$, thus we have

$$
V\left(t, x_{t}\right) \leq V\left(t_{k-1}, x_{t_{k-1}}\right) e^{c\left(t-t_{k-1}\right)}, \quad t \in\left[t_{k-1}, t_{k}\right), k \in \mathbb{N}^{*} .
$$

Then, we obtain that, for $t \in\left[t_{0}, t_{1}\right)$,

$$
\begin{aligned}
V\left(t, x_{t}\right) & \leq V\left(t_{0}, x_{t_{0}}\right) e^{c\left(t-t_{0}\right)} \\
& \leq\left[\left\|x\left(t_{0}\right)\right\|+\max _{1 \leq j \leq n}\left\{\sum_{i=1}^{n}\left|b_{i j}\right| L_{j}\right\} \tau\left\|\phi-u^{*}\right\|_{\tau}\right] e^{c\left(t-t_{0}\right)} \\
& \leq(1+\lambda \tau)\left\|\phi-u^{*}\right\|_{\tau} e^{c\left(t_{1}-t_{0}\right)}
\end{aligned}
$$

where $\lambda=\max _{1 \leq j \leq n}\left\{\sum_{i=1}^{n}\left|b_{i j}\right| L_{j}\right\},\|x(t)\|=\sum_{i=1}^{n}\left|x_{i}\right|$. Then

$$
\|x(t)\| \leq V\left(t, x_{t}\right) \leq M\left\|\phi-u^{*}\right\|_{\tau} e^{-\alpha\left(t-t_{0}\right)}, \quad t \in\left[t_{0}, t_{1}\right),
$$

where $M=(1+\lambda \tau) e^{(c+\alpha) l}$. So from (3.2), we have

$$
\begin{aligned}
&\left\|x\left(t_{1}^{-}\right)\right\| \leq(1+\lambda \tau)\left\|\phi-u^{*}\right\|_{\tau} e^{c\left(t_{1}-t_{0}\right)}, \\
&\left\|x_{t_{1}^{-}}\right\|_{\tau} \leq(1+\lambda \tau)\left\|\phi-u^{*}\right\|_{\tau} e^{c\left(t_{1}-t_{0}\right)} .
\end{aligned}
$$

Therefore we obtain

$$
\begin{aligned}
V\left(t_{1}, x_{t_{1}}\right) & =\sum_{i=1}^{n}\left(\left|x_{i}\left(t_{1}\right)\right|+\sum_{j=1}^{n}\left|b_{i j}\right| L_{j} \int_{t_{1}-\tau_{j}}^{t_{1}}\left|x_{j}(s)\right| d s\right) \\
& \leq d\left\|x\left(t_{1}^{-}\right)\right\|+\sum_{i=1}^{n} \sum_{j=1}^{n}\left|b_{i j}\right| L_{j}\left|x_{j_{1}^{-}}\right|_{\tau} \tau_{j} \\
& \leq d\left\|x\left(t_{1}^{-}\right)\right\|+\tau \lambda\left\|x_{t_{1}^{-}}\right\|_{\tau} \leq(d+\lambda \tau)\left\|x_{t_{1}^{-}}\right\|_{\tau} \\
& \leq(d+\lambda \tau)(1+\lambda \tau)\left\|\phi-u^{*}\right\|_{\tau} e^{c\left(t_{1}-t_{0}\right)} \\
& \leq e^{-(c+\alpha) l} M\left\|\phi-u^{*}\right\|_{\tau} e^{-\alpha l}
\end{aligned}
$$

thus we have, for $t \in\left[t_{1}, t_{2}\right)$

$$
\begin{aligned}
V\left(t, x_{t}\right) & \leq V\left(t_{1}, x_{t_{1}}\right) e^{c\left(t-t_{1}\right)} \leq V\left(t_{1}, x_{t_{1}}\right) e^{c l} \\
& \leq e^{-2 \alpha l} M\left\|\phi-u^{*}\right\|_{\tau} \\
& \leq M\left\|\phi-u^{*}\right\|_{\tau} e^{-\alpha\left(t-t_{0}\right)}
\end{aligned}
$$

and hence we get

$$
\|x(t)\| \leq M\left\|\phi-u^{*}\right\|_{\tau} e^{-\alpha\left(t-t_{0}\right)}, \quad t \in\left[t_{1}, t_{2}\right) .
$$


Next we shall show that

$$
V\left(t_{i}, x_{t_{i}}\right) \leq e^{-(i+1) \alpha l} e^{-c l} M\left\|\phi-u^{*}\right\|_{\tau}, \quad i \in \mathbb{N}^{*} .
$$

We know (3.6) holds for $i=1$ in view of (3.5), if we assume that it holds for $i=k$, i.e.

$$
V\left(t_{k}, x_{t_{k}}\right) \leq e^{-(k+1) \alpha l} e^{-c l} M\left\|\phi-u^{*}\right\|_{\tau}, \quad k \in \mathbb{N}^{*},
$$

then we have, for $t \in\left[t_{k}, t_{k+1}\right)$

$$
\begin{aligned}
V\left(t, x_{t}\right) & \leq V\left(t_{k}, x_{t_{k}}\right) e^{c\left(t-t_{k}\right)} \leq V\left(t_{k}, x_{t_{k}}\right) e^{c l} \\
& \leq e^{-(k+1) \alpha l} M\left\|\phi-u^{*}\right\|_{\tau}
\end{aligned}
$$

and

$$
\begin{gathered}
\|x(t)\| \leq V\left(t, x_{t}\right) \leq e^{-(k+1) \alpha l} M\left\|\phi-u^{*}\right\|_{\tau}, \\
\left\|x_{t_{k+1}^{-}}\right\|_{\tau} \leq V\left(t, x_{t}\right) \leq e^{-(k+1) \alpha l} M\left\|\phi-u^{*}\right\|_{\tau},
\end{gathered}
$$

therefore we obtain

$$
\begin{aligned}
V\left(t_{k+1}, x\left(t_{k+1}\right)\right) & =\sum_{i=1}^{n}\left[\left|x_{i}\left(t_{k+1}\right)\right|+\sum_{j=1}^{n}\left|b_{i j}\right| L_{j}\left|\int_{t_{k+1}-\tau_{j}}^{t_{k+1}}\right| x_{j}(s) \mid d s\right] \\
& \leq d\left\|x\left(t_{k+1}^{-}\right)\right\|+\lambda \tau\left\|x_{t_{k+1}^{-}}\right\|_{\tau} \\
& \leq(d+\lambda \tau) e^{-(k+1) \alpha l} M\left\|\phi-u^{*}\right\|_{\tau} \\
& \leq e^{-(\alpha+c) l} e^{-(k+1) \alpha l} M\left\|\phi-u^{*}\right\|_{\tau} \\
& \leq e^{-(k+2) \alpha l} e^{-c l} M\left\|\phi-u^{*}\right\|_{\tau}
\end{aligned}
$$

which implies that (3.6) holds for $i=k+1$, and hence (3.6) holds for any $i \in \mathbb{N}^{*}$. So we have

$$
\begin{aligned}
\|x(t)\| & \leq V(t, x(t)) \leq V\left(t_{i}, x\left(t_{i}\right)\right) e^{c\left(t-t_{i}\right)} \\
& \leq e^{-(i+1) \alpha l} M\left\|\phi-u^{*}\right\|_{\tau} \\
& \leq M\left\|\phi-u^{*}\right\|_{\tau} e^{-\alpha\left(t-t_{0}\right)}, \quad t \in\left[t_{i}, t_{i+1}\right), i \in \mathbb{N}^{*}
\end{aligned}
$$

which, together with (3.3), yields the global exponential stability of $u^{*}$.

If we change the proof in Theorem $3.1 \mathrm{a}$ bit, then we will have the following result in which the lower bound of the length of the successive impulses is relaxed but the restriction on the amplitude of impulses is stronger than in Theorem 3.1

Theorem 3.2. Assume that there exist constants $l, \alpha, d>0$ such that $t_{k}-t_{k-1} \leq$ $l,\left|I_{i}\left(y+u_{i}^{*}\right)+y\right| \leq d|y|$ for any $y \in \mathbb{R}$ with

$$
\ln \left(d+\lambda \tau e^{\alpha \tau}\right) \leq-(\alpha+c) l
$$

where $c=\max _{1 \leq i \leq n}\left\{-c_{i}+\sum_{j=1}^{n}\left(\left|a_{j i}\right|+\left|b_{j i}\right|\right) L_{i}\right\}>0$, and $\lambda=\max _{1 \leq j \leq n}\left\{\sum_{i=1}^{n}\right.$ $\left.\left|b_{i j}\right| L_{j}\right\}$, then the equilibrium point $u=u^{*}$ of system (2.1) is globally exponentially stable. 
Proof. By choosing the same Lyapunov functional and using the same argument as in Theorem 3.1, we get $V^{\prime}\left(t, x_{t}\right) \leq c V\left(t, x_{t}\right)$ for $t \in\left[t_{k-1}, t_{k}\right)$ and $k \in \mathbb{N}^{*}$ and $\|x(t)\| \leq M\left\|\phi-u^{*}\right\|_{\tau} e^{-\alpha\left(t-t_{0}\right)}$ for $t \in\left[t_{0}, t_{1}\right)$. Next we shall show by mathematical induction that

$$
\|x(t)\| \leq M\left\|\phi-u^{*}\right\|_{\tau} e^{-\alpha\left(t-t_{0}\right)}, \quad t \in\left[t_{i-1}, t_{i}\right), \quad i \in \mathbb{N}^{*} .
$$

We have that (3.7) holds for $i=1$ by the same argument in Theorem 3.1, and then we assume it holds for $i=k$, i.e.

$$
\|x(t)\| \leq M\left\|\phi-u^{*}\right\|_{\tau} e^{-\alpha\left(t-t_{0}\right)}, \quad t \in\left[t_{k-1}, t_{k}\right),
$$

we shall show that (3.7) holds for $i=k+1$ :

$$
\|x(t)\| \leq M\left\|\phi-u^{*}\right\|_{\tau} e^{-\alpha\left(t-t_{0}\right)}, \quad t \in\left[t_{k}, t_{k+1}\right) .
$$

From (3.8), we get

$$
\left\|x\left(t_{k}^{-}\right)\right\| \leq M\left\|\phi-u^{*}\right\|_{\tau} e^{-\alpha\left(t_{k}-t_{0}\right)}
$$

and

$$
\left\|x_{t_{k}^{-}}\right\|_{\tau} \leq M\left\|\phi-u^{*}\right\|_{\tau} e^{\alpha \tau} e^{-\alpha\left(t_{k}-t_{0}\right)}
$$

and hence

$$
\begin{aligned}
V\left(t_{k}, x_{t_{k}}\right) & =\sum_{i=1}^{n}\left[\left|x_{i}\left(t_{k}\right)\right|+\sum_{j=1}^{n}\left|b_{i j}\right| L_{j} \int_{t_{k}-\tau_{j}}^{t_{k}}\left|x_{j}(s) d s\right|\right] \\
& \leq \sum_{i=1}^{n}\left[d\left|x_{i}\left(t_{k}^{-}\right)\right|+\sum_{j=1}^{n}\left|b_{i j}\right| L_{j} \int_{t_{k}^{-}-\tau_{j}}^{t_{k}^{-}}\left|x_{j}(s) d s\right|\right] \\
& \leq d\left\|x\left(t_{k}^{-}\right)\right\|+\lambda \tau\left\|x_{t_{k}^{-}}\right\|_{\tau} \\
& \leq M\left(d+\lambda \tau e^{\alpha \tau}\right)\left\|\phi-u^{*}\right\|_{\tau} e^{-\alpha\left(t_{k}-t_{0}\right)} \\
& \leq e^{-(\alpha+c) l} M\left\|\phi-u^{*}\right\|_{\tau} e^{-\alpha\left(t_{k}-t_{0}\right)}
\end{aligned}
$$

then for $t \in\left[t_{k}, t_{k+1}\right)$, we have

$$
\begin{aligned}
V\left(t, x_{t}\right) & \leq V\left(t_{k}, x_{t_{k}}\right) e^{c\left(t-t_{k}\right)} \leq V\left(t_{k}, x_{t_{k}}\right) e^{c l} \\
& \leq e^{-\alpha l} M\left\|\phi-u^{*}\right\|_{\tau} e^{-\alpha\left(t_{k}-t_{0}\right)} \\
& \leq M\left\|\phi-u^{*}\right\|_{\tau} e^{-\alpha\left(t-t_{0}\right)}
\end{aligned}
$$

this gives

$$
\|x(t)\| \leq V\left(t, x_{t}\right) \leq M\left\|\phi-u^{*}\right\|_{\tau} e^{-\alpha\left(t-t_{0}\right)}, \quad t \in\left[t_{k}, t_{k+1}\right),
$$

which implies (3.7) holds for all $t \geq t_{0}$ and completes the proof.

When $c$ is non-positive, the method in Theorem 3.1 can not apply, by using similar method in Theorem 3.2 , we can obtain the result as follows.

Theorem 3.3. Assume that there exist constants $\alpha, d>0$ such that $\max _{1 \leq i \leq n}$ $\left\{-c_{i}+\sum_{j=1}^{n}\left(\left|a_{j i}\right|+\left|b_{j i}\right|\right) L_{i}\right\} \triangleq c \leq 0$ and $\left|I_{i}\left(y+u_{i}^{*}\right)+y\right| \leq d|y|$ for any $y \in \mathbb{R}$ with

$$
\ln \left(d+\lambda \tau e^{\alpha \tau}\right) \leq-\alpha\left(t_{k}-t_{k-1}\right), \quad k \in \mathbb{N}^{*}
$$


where $\lambda=\max _{1 \leq j \leq n}\left\{\sum_{i=1}^{n}\left|b_{i j}\right| L_{j}\right\}$, then the equilibrium point $u=u^{*}$ of system (2.1) is globally exponentially stable.

Proof. Choosing the same Lyapunov functional and using the same argument as in Theorem 3.1, we get $V^{\prime}\left(t, x_{t}\right) \leq c V\left(t, x_{t}\right)$ for $t \in\left[t_{k-1}, t_{k}\right)$. Since $c \geq 0$, we have $V^{\prime}\left(t, x_{t}\right) \leq 0$ for $t \geq t_{0}$. Then similarly to Theorem 3.1, we have

$$
\begin{aligned}
\|x(t)\| & \leq V\left(t, x_{t}\right) \leq V\left(t_{0}, x_{t_{0}}\right) \\
& \leq(1+\lambda \tau)\left\|\phi-u^{*}\right\|_{\tau} \\
& \leq M\left\|\phi-u^{*}\right\|_{\tau} e^{-\alpha\left(t-t_{0}\right)}, \quad t \in\left[t_{0}, t_{1}\right),
\end{aligned}
$$

where $M=(1+\lambda \tau) e^{c l}$. And hence we have

$$
\left\|x\left(t_{1}^{-}\right)\right\| \leq M\left\|\phi-u^{*}\right\|_{\tau} e^{-\alpha\left(t_{1}-t_{0}\right)}
$$

and

$$
\left\|x_{t_{1}^{-}}\right\|_{\tau} \leq M\left\|\phi-u^{*}\right\|_{\tau} e^{\alpha \tau} e^{-\alpha\left(t_{1}-t_{0}\right)}
$$

Then for $t \in\left[t_{1}, t_{2}\right)$, we obtain

$$
\begin{aligned}
V\left(t, x_{t}\right) & \leq V\left(t_{1}, x_{t_{1}}\right) \leq d\left\|x\left(t_{1}^{-}\right)\right\|+\lambda \tau\left\|x_{t_{1}^{-}}\right\|_{\tau} \\
& \leq\left(d+\lambda \tau e^{\alpha \tau}\right) M\left\|\phi-u^{*}\right\|_{\tau} e^{-\alpha\left(t_{1}-t_{0}\right)} \\
& \leq e^{-\alpha\left(t_{2}-t_{1}\right)} M\left\|\phi-u^{*}\right\|_{\tau} e^{-\alpha\left(t_{1}-t_{0}\right)} \\
& \leq M\left\|\phi-u^{*}\right\|_{\tau} e^{-\alpha\left(t-t_{0}\right)}
\end{aligned}
$$

that gives

$$
x(t) \leq V\left(t, x_{t}\right) \leq M\left\|\phi-u^{*}\right\|_{\tau} e^{-\alpha\left(t-t_{0}\right)}, \quad t \in\left[t_{1}, t_{2}\right) .
$$

Similarly, we can prove that

$$
x(t) \leq V\left(t, x_{t}\right) \leq M\left\|\phi-u^{*}\right\|_{\tau} e^{-\alpha\left(t-t_{0}\right)}, \quad t \in\left[t_{k-1}, t_{k}\right), k \in \mathbb{N}^{*},
$$

which implies our result.

By using the same method in Theorem 3.1 and different Lyapunov functional, we have the following result.

Theorem 3.4. Assume that there exist constants $l, \alpha, d>0$ such that $\tau \leq$ $t_{k}-t_{k-1} \leq l,\left|I_{i}\left(y+u_{i}^{*}\right)+y\right| \leq d|y|$ for any $y \in \mathbb{R}$ with

$$
\ln \left(d^{2}+\lambda \tau\right) \leq-(c+2 \alpha) l,
$$

where $c=\max _{1 \leq i \leq n}\left\{-2 c_{i}+\sum_{j=1}^{n}\left(\left|a_{j i}\right| L_{i}+\left|a_{i j}\right| L_{j}+\left|b_{j i}\right| L_{i}+\left|b_{i j}\right| L_{j}\right)\right\}>0$, and $\lambda=\max _{1 \leq j \leq n}\left\{\sum_{i=1}^{n}\left|b_{i j}\right| L_{j}\right\}$, then the equilibrium point $u=u^{*}$ of system (2.1) is globally exponentially stable. 
Proof. Choose Lyapunov functional

$$
V\left(t, x_{t}\right)=\sum_{i=1}^{n}\left[x_{i}^{2}(t)+\sum_{j=1}^{n}\left|b_{i j}\right| L_{j} \int_{t-\tau_{j}}^{t} x_{j}^{2}(s) d s\right],
$$

then the Dini derivative of $V$ along system $(\overline{2.5})$ is

$$
\begin{array}{ll}
\frac{d V\left(t, x_{t}\right)}{d t} \quad=\sum_{i=1}^{n}\left[2 x _ { i } ( t ) \left\{-c_{i} x_{i}(t)+\sum_{j=1}^{n} a_{i j}\left(f_{j}\left(x_{j}(t)+u_{j}^{*}\right)-f_{j}\left(u_{j}^{*}\right)\right)+\sum_{j=1}^{n} b_{i j}\right.\right. \\
\left.\left.\times \quad\left(f_{j}\left(x_{j}\left(t-\tau_{j}\right)+u_{j}^{*}\right)-f_{j}\left(u_{j}^{*}\right)\right)\right\}+\sum_{j=1}^{n}\left|b_{i j}\right| L_{j}\left(x_{j}^{2}(t)-x_{j}^{2}\left(t-\tau_{j}\right)\right)\right] \\
\leq \quad \sum_{i=1}^{n}\left[-2 c_{i} x_{i}^{2}(t)+\sum_{j=1}^{n} 2\left|x_{i}(t)\right|\left|a_{i j}\right| L_{j}\left|x_{j}(t)\right|+\sum_{j=1}^{n} 2\left|x_{i}(t)\right|\left|b_{i j}\right| L_{j}\right. \\
\left.\times \quad\left|x_{j}\left(t-\tau_{j}\right)\right|+\sum_{j=1}^{n}\left|b_{i j}\right| L_{j}\left(\left|x_{j}(t)\right|^{2}-\left|x_{j}\left(t-\tau_{j}\right)\right|^{2}\right)\right] \\
\leq \quad-2 \sum_{i=1}^{n} c_{i} x_{i}^{2}(t)+\sum_{i=1}^{n} \sum_{j=1}^{n}\left|a_{i j}\right| L_{j}\left(\left|x_{i}(t)\right|^{2}+\left|x_{j}(t)\right|^{2}\right)+\sum_{i=1}^{n} \sum_{j=1}^{n}\left|b_{i j}\right| L_{j} \\
\times \quad\left(\left|x_{i}(t)\right|^{2}+\left|x_{j}\left(t-\tau_{j}\right)\right|^{2}\right)+\sum_{i=1}^{n} \sum_{j=1}^{n}\left|b_{i j}\right| L_{j}\left(\left|x_{j}(t)\right|^{2}-\left|x_{j}\left(t-\tau_{j}\right)\right|^{2}\right) \\
\leq \quad \sum_{i=1}^{n}\left[-2 c_{i}+\sum_{j=1}^{n}\left(\left|a_{j i}\right| L_{i}+\left|a_{i j}\right| L_{j}+\left|b_{j i}\right| L_{i}+\left|b_{i j}\right| L_{j}\right)\right]\left|x_{i}(t)\right|^{2} \\
\leq \quad \mathrm{c}\left(t, x_{t}\right), \quad t \in\left[t_{k-1}, t_{k}\right), k \in \mathbb{N}^{*},
\end{array}
$$

where $c=\max _{1 \leq i \leq n}\left\{-2 c_{i}+\sum_{j=1}^{n}\left(\left|a_{j i}\right| L_{i}+\left|a_{i j}\right| L_{j}+\left|b_{j i}\right| L_{i}+\left|b_{i j}\right| L_{j}\right)\right\}>0$, thus we have

$$
V\left(t, x_{t}\right) \leq V\left(t_{k-1}, x_{t_{k-1}}\right) e^{c\left(t-t_{k-1}\right)}, \quad t \in\left[t_{k-1}, t_{k}\right), k \in \mathbb{N}^{*} .
$$

Then, we obtain that, for $t \in\left[t_{0}, t_{1}\right)$,

$$
\begin{aligned}
V\left(t, x_{t}\right) & \leq V\left(t_{0}, x_{t_{0}}\right) e^{c\left(t-t_{0}\right)} \\
& \left.\leq\left[\left\|x\left(t_{0}\right)\right\|^{2}+\max _{1 \leq j \leq n}\left\{\sum_{i=1}^{n}\left|b_{i j}\right| L_{j}\right\} \tau\left\|\phi-u^{*}\right\|_{\tau}\right)^{2}\right] e^{c\left(t-t_{0}\right)} \\
& \leq(1+\lambda \tau)\left\|\phi-u^{*}\right\|_{\tau}^{2} e^{c\left(t_{1}-t_{0}\right)}
\end{aligned}
$$

where $\lambda=\max _{1 \leq j \leq n}\left\{\sum_{i=1}^{n}\left|b_{i j}\right| L_{j}\right\},\|x(t)\|=\left(\sum_{i=1}^{n}\left|x_{i}\right|^{2}\right)^{\frac{1}{2}}$. Then

$$
\|x(t)\| \leq \sqrt{V\left(t, x_{t}\right)} \leq M\left\|\phi-u^{*}\right\|_{\tau} e^{-\alpha\left(t-t_{0}\right)}, \quad t \in\left[t_{0}, t_{1}\right),
$$

where $M=\sqrt{1+\lambda \tau} e^{\left(\frac{c}{2}+\alpha\right) l}$. So from (3.10) and (3.11), we have

$$
\begin{aligned}
\left\|x\left(t_{1}^{-}\right)\right\|^{2} & \leq(1+\lambda \tau)\left\|\phi-u^{*}\right\|_{\tau}^{2} e^{c\left(t_{1}-t_{0}\right)} \\
\left\|x_{t_{1}^{-}}\right\|_{\tau}^{2} & \leq(1+\lambda \tau)\left\|\phi-u^{*}\right\|_{\tau}^{2} e^{c\left(t_{1}-t_{0}\right)}
\end{aligned}
$$


Therefore we obtain

$$
\begin{aligned}
V\left(t_{1}, x_{t_{1}}\right) & =\sum_{i=1}^{n}\left(\left|x_{i}\left(t_{1}\right)\right|^{2}+\sum_{j=1}^{n}\left|b_{i j}\right| L_{j} \int_{t_{1}-\tau_{j}}^{t_{1}}\left|x_{j}(s)\right|^{2} d s\right) \\
& \leq d^{2}\left\|x_{1}\left(t_{1}^{-}\right)\right\|^{2}+\sum_{i=1}^{n} \sum_{j=1}^{n}\left|b_{i j}\right| L_{j}\left|x_{j_{1}}\right|_{\tau}^{2} \tau_{j} \\
& \leq d^{2}\left\|x_{1}\left(t_{1}^{-}\right)\right\|^{2}+\tau \lambda\left\|x_{t_{1}^{-}}\right\|_{\tau}^{2} \leq\left(d^{2}+\lambda \tau\right)\left\|x_{t_{1}^{-}}\right\|_{\tau}^{2} \\
& \leq\left(d^{2}+\lambda \tau\right)(1+\lambda \tau)\left\|\phi-u^{*}\right\|_{\tau}^{2} e^{c\left(t_{1}-t_{0}\right)} \\
& \leq e^{-(c+2 \alpha) l} M^{2}\left\|\phi-u^{*}\right\|_{\tau}^{2} e^{-2 \alpha l}
\end{aligned}
$$

thus we have, for $t \in\left[t_{1}, t_{2}\right)$

$$
\begin{aligned}
V\left(t, x_{t}\right) & \leq V\left(t_{1}, x_{t_{1}}\right) e^{c\left(t-t_{1}\right)} \leq V\left(t_{1}, x_{t_{1}}\right) e^{c l} \\
& \leq e^{-4 \alpha l} M^{2}\left\|\phi-u^{*}\right\|_{\tau}^{2} \\
& =M^{2}\left\|\phi-u^{*}\right\|_{\tau}^{2} e^{-2 \alpha\left(t-t_{0}\right)}
\end{aligned}
$$

and hence we get

$$
\|x(t)\| \leq M\left\|\phi-u^{*}\right\|_{\tau} e^{-\alpha\left(t-t_{0}\right)}, \quad t \in\left[t_{1}, t_{2}\right) .
$$

Similarly to Theorem 3.1, we can derive

$$
\|x(t)\| \leq M\left\|\phi-u^{*}\right\|_{\tau} e^{-\alpha\left(t-t_{0}\right)}, \quad t \geq t_{0},
$$

which completes the proof.

Remark 3.1. Compared to Theorem 3.1, the result in Theorem 3.4, if both theorems are applicable (i.e. $c>0$ ), seems less restrictive for small value of $\alpha$ and $l$, hence we expect a bigger bound on the impulse amplitude d from Theorem 3.4 in the stability analysis of the same problem; however, for big value of c, either the difference is small or we can obtain a bigger bound on the impulse amplitude d from Theorem 3.1, see Example 4.1.

Using the same Lyapunov functional as in Theorem 3.4 and similar method in Theorem 3.2 and Theorem 3.4 , we have the following result.

Theorem 3.5. Assume that there exist constants $l, \alpha, d>0$ such that $t_{k}-t_{k-1} \leq$ $l,\left|I_{i}\left(y+u_{i}^{*}\right)+y\right| \leq d|y|$ for any $y \in \mathbb{R}$ with

$$
\ln \left(d^{2}+\lambda \tau e^{2 \alpha \tau}\right) \leq-(c+2 \alpha) l
$$

where $c=\max _{1 \leq i \leq n}\left\{-2 c_{i}+\sum_{j=1}^{n}\left(\left|a_{j i}\right| L_{i}+\left|a_{i j}\right| L_{j}+\left|b_{j i}\right| L_{i}+\left|b_{i j}\right| L_{j}\right)\right\}>0, \lambda=$ $\max _{1 \leq j \leq n}\left\{\sum_{i=1}^{n}\left|b_{i j}\right| L_{j}\right\}$, then the equilibrium point $u=u^{*}$ of system (2.1) is globally exponentially stable.

Using the same Lyapunov functional as in Theorem 3.4 and similar method to Theorem 3.3 and Theorem 3.4, we have the following result. 
Theorem 3.6. Assume that there exist constants $\alpha, d>0$ such that $\max _{1 \leq i \leq n}$ $\left\{-2 c_{i}+\sum_{j=1}^{n}\left(\left|a_{j i}\right| L_{i}+\left|a_{i j}\right| L_{j}+\left|b_{j i}\right| L_{i}+\left|b_{i j}\right| L_{j}\right)\right\} \triangleq c \leq 0$ and $\left|I_{i}\left(y+u_{i}^{*}\right)+y\right| \leq d|y|$ for any $y \in \mathbb{R}$ with

$$
\ln \left(d^{2}+\lambda \tau e^{2 \alpha \tau}\right) \leq-\alpha\left(t_{k}-t_{k-1}\right), \quad k \in \mathbb{N}^{*}
$$

where $\lambda=\max _{1 \leq j \leq n}\left\{\sum_{i=1}^{n}\left|b_{i j}\right| L_{j}\right\}$, then the equilibrium point $u=u^{*}$ of system (2.1) is globally exponentially stable.

Remark 3.2. Notice that the cellular neural networks without impulses might be stable when $c \leq 0$ in Theorem 3.6 and Theorem 3.3, see Example 4.2.

\section{EXAmples}

In this section, we will discuss some examples to illustrate our results, simulations are also given to make the results more intuitive.

Example 4.1. Consider the following cellular neural networks with time delay

$$
\left\{\begin{aligned}
\frac{d u_{1}(t)}{d t}= & -u_{1}(t)+\frac{1}{2} f\left(u_{1}(t)\right)+\frac{1}{2} f\left(u_{2}(t)\right)-\frac{3}{2} f\left(u_{1}(t-0.01)\right) \\
& -\frac{3}{2} f\left(u_{2}(t-0.075)\right)+2 \\
\frac{d u_{2}(t)}{d t}= & -0.5 u_{2}(t)+\frac{1}{2} f\left(u_{1}(t)\right)+f\left(u_{2}(t)\right)-f\left(u_{1}(t-0.01)\right) \\
& -\frac{1}{2} f\left(u_{2}(t-0.075)\right)+2, \quad t \geq 0 \\
= & \phi
\end{aligned}\right.
$$

where $f(s)=\frac{1}{2}(|s+1|-|s-1|)$ for any $s \in \mathbb{R}, \phi \in P C\left([-0.01,0], \mathbb{R}^{2}\right)$.

By direct computation, we know that $u^{*}=(0.5,4.5)$ is the unique equilibrium point of the cellular neural networks (4.1).

It is easy to check that the condition in Corollary 3 ([5]) does not hold since

$$
\begin{aligned}
& c_{1}<\left|a_{11}\right|+\left|a_{12}\right|+\left|b_{11}\right|+\left|b_{12}\right| ; \\
& c_{2}<\left|a_{21}\right|+\left|a_{22}\right|+\left|b_{21}\right|+\left|b_{22}\right|,
\end{aligned}
$$

and the condition in [2] is not satisfied since the matrix $-\left(A+A^{T}\right)$ is not positive definite, so the equilibrium point $u^{*}$ might not be exponentially stable. Actually, the numerical simulation shows that $u^{*}$ is not even asymptotically stable, see Fig.1 for graphs of the solutions with different initial functions $\phi=(-0.1 H(t), 4 H(t))^{T}$, $(2 H(t), 6 H(t))^{T},(6 H(t),-5 H(t))^{T}$, where $H(t)$ is the Heaviside step function. 


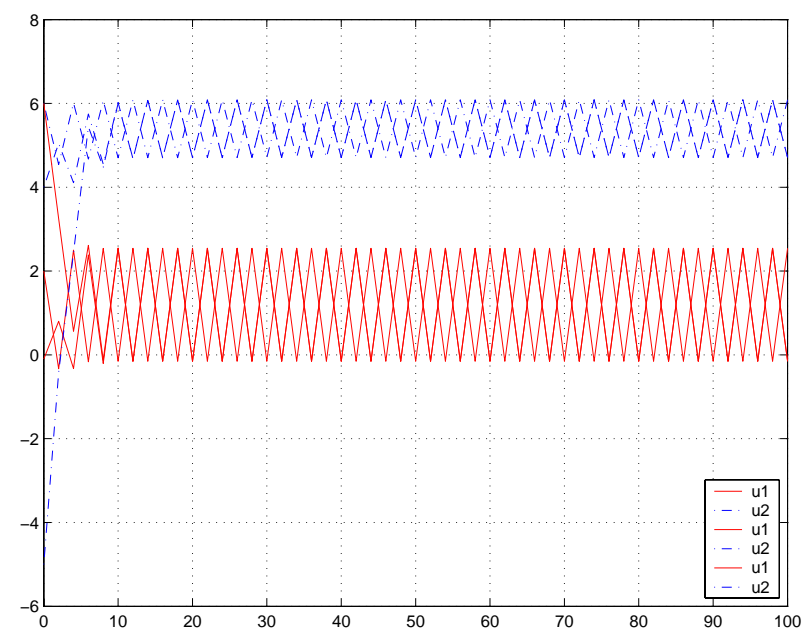

Fig.1. System without impulses.

In Theorem 3.1, it can easily check that $c=3>0, \lambda=\frac{5}{2}, \tau=0.01$; if we choose $\alpha=0.1, l=0.5$, then by Theorem 3.1, we can obtain the estimate for the impulse amplitude which can stabilize this cellular neural network is

$$
d \leq e^{-(\alpha+c) l}-\lambda \tau \leq 0.1872
$$

Thus we can choose the impulse control functions $I_{i}(s)=-0.85 s+0.85 u_{i}^{*}$ for any $s \in \mathbb{R}$ to stabilize cellular neural network (4.1), see Fig.2 for the numerical simulations with the same initial functions as in Fig.1.

However, if we use Theorem 3.4 in stead, we obtain $c=5.5>0$ and the estimate for the impulse amplitude which can stabilize this cellular neural network is

$$
d \leq \sqrt{e^{-(2 \alpha+c) l}-\lambda \tau} \leq 0.1669
$$

which allows a smaller bound for the impulse amplitude; while if we choose smaller $l$, for example, $l=0.25$ in both theorems, then from Theorem 3.4 we obtain $d \leq 0.4588$, which is a bigger bound for the amplitude of impulsive control than the result $d \leq 0.4357$ from Theorem 3.1. This verifies the prediction in Remark 3.1 . 


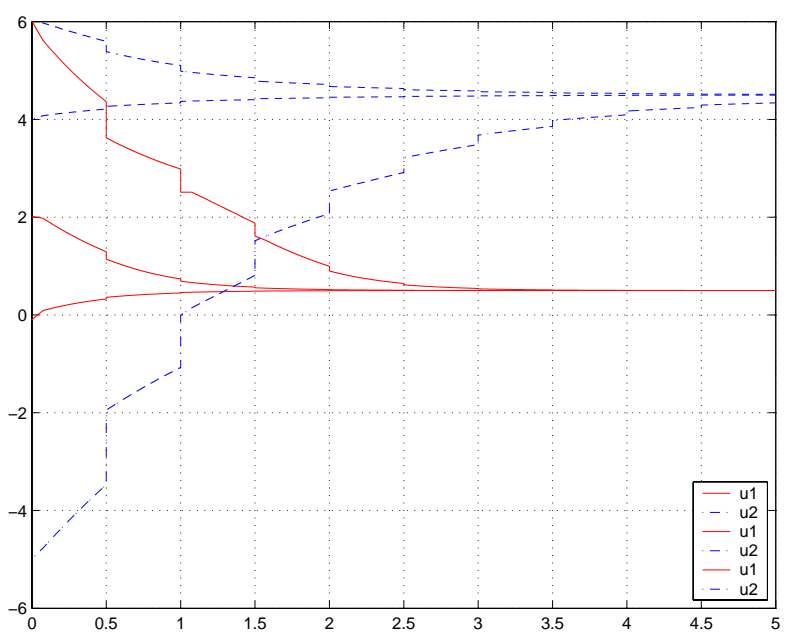

Fig.2. System with impulsive control.

In the above example, we show that how Theorem 3.1 and Theorem 3.4 work in stabilizing the unstable neural network (4.1). Next we shall try to use Theorem 3.3 to determine the stability of neural networks. In fact, when $c \leq 0$, the neural network without impulses might be stable, as the following example indicates.

Example 4.2. Consider the following cellular neural networks with time delay

$$
\left\{\begin{aligned}
\frac{d u_{1}(t)}{d t}= & -2 u_{1}(t)+\frac{2}{3} f\left(u_{1}(t)\right)+\frac{1}{5} f\left(u_{2}(t)\right)+\frac{4}{5} f\left(u_{1}(t-0.01)\right) \\
& +\frac{1}{3} f\left(u_{2}(t-0.075)\right)+3 \\
\frac{d u_{2}(t)}{d t}= & -2 u_{2}(t)+\frac{1}{3} f\left(u_{1}(t)\right)+\frac{4}{5} f\left(u_{2}(t)\right)+\frac{1}{5} f\left(u_{1}(t-0.01)\right) \\
& +\frac{2}{3} f\left(u_{2}(t-0.075)\right)+2.5, \quad t \geq 0 \\
= & \phi
\end{aligned}\right.
$$

where $f(s)=\frac{1}{2}(|s+1|-|s-1|)$ for any $s \in \mathbb{R}, \phi \in P C\left([-0.01,0], \mathbb{R}^{2}\right)$.

By direct computation, we know that $u^{*}=(2.5,2.25)$ is the unique equilibrium point of the cellular neural networks (4.2). Moreover, $c_{i}=2=\sum_{j=1}^{n}\left(\left|a_{j i}\right|+\right.$ $\left.\left|b_{j i}\right|\right) L_{i}=\frac{1}{2} \sum_{j=1}^{n}\left(\left|a_{j i}\right| L_{i}+\left|a_{i j}\right| L_{j}+\left|b_{j i}\right| L_{i}+\left|b_{i j}\right| L_{j}\right)$, which yields $c=0$ in Theorem 3.3 and Theorem 3.6, the simulations, with initial functions

$$
\phi=(-5 H(t), 5 H(t))^{T},(2 H(t), 3 H(t))^{T},(3 H(t), 2 H(t))^{T}
$$

(see Fig.3), show that the equilibrium point $u^{*}$ of this system might be globally exponentially stable. 


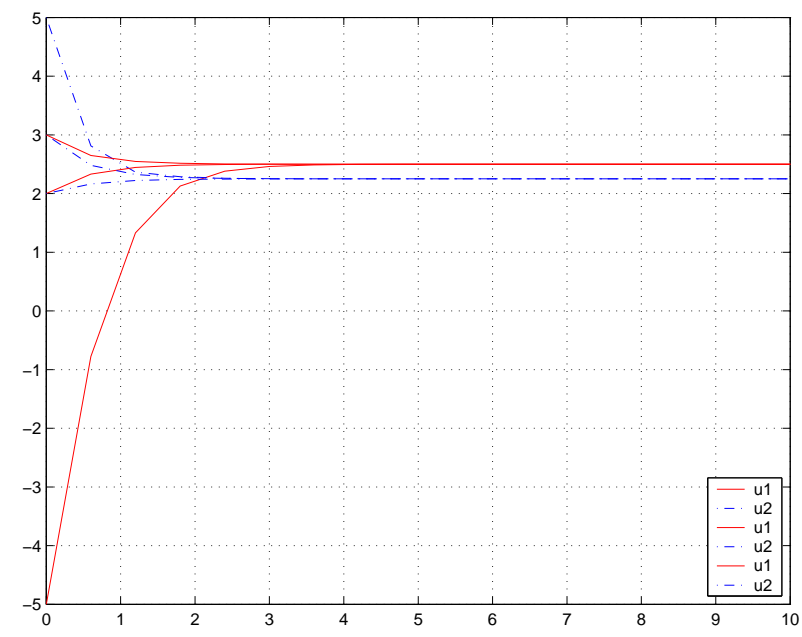

Fig.3. Stable system when $c=0$.

\section{Conclusions}

In this paper, some global exponential stability criteria have been established for impulsive cellular neural networks with tim delay based on Lyapunov functional method. Our results show that impulses may play an important role in the stabilization of some cellular neural networks with time delay. The conditions are easy to verify. The discussions above assume constant delays, cellular neural networks with time-varying delays will be investigated in the near future.

Acknowledgements: This research was supported by NSERC-Canada which is gratefully acknowledged.

\section{REFERENCES}

[1] H. Akca, R. Alassar, V. Covachev, Z. Covacheva and E. Al-Zahrani, Continuous-time additive Hopfield-type neural networks with impulses, J. Math. Anal. Appl., 290(2004), 436-451. 1

[2] S. Arik and V.Tavsanglu, On the global asymptotic stability of delayed cellular neural networks, IEEE Trans. Circuits Syst. I, 47(2000), 571-574. 1, 4

[3] G. Ballinger and X. Liu, Existence and uniqueness results for impulsive delay differential equations, Dynamics of Continuous, Discrete and Impulsive Systems, 5(1999), 579-591. 2

[4] J. Cao, J. Liang, J. Lam, Exponential stability of high-order bidirectional associative memory neural networks with time delays, Physica D, 199(2004), 425-436. 1

[5] J. Cao and J. Wang, Global Exponential Stability and Periodicity of Recurrent Neural Networks with Time Delays, IEEE Trans Circuits Syst. I, 52(2005), 920-931. 1, 4 
[6] L. Chua and T. Roska, Cellular neural networks with nonlinear and delay-type template elements, Proceeding of 1990 IEEE Int. Workshop Cellular Neural Networks Applications, 12-25. 1

[7] L. Chua and T. Roska, Stability of a class of nonreciprocal cellular neural networks, IEEE Trans. Circuits Syst., 37(1990), 1520-1527. 1

[8] L. Chua and L. Yang, Cellular neural networks: theory, IEEE Trans. Circuits Syst., 35(1988), 1257-1271. 1

[9] P. Civalleri, M. Gilli, and L. Pandolfi, On stability of cellular neural networks with delay, IEEE Trans. Circuits Syst. I, 40(1993), 157-165. 1

[10] K. Gopalsamy, Stability of artificial neural networks with impulses, Applied Mathematics and Computation, 154(2004), 783-813. 1

[11] X. Li, L. Huang and J. Wu, Further results on the stability of delayed cellular neural networks, IEEE Trans. Circuits Syst. I, 50(2003), 1239-1242. 1

[12] X. Liu and Q. Wang, Exponential Stability of Impulsive Functional Differential Equations via Lyapunov Functionals, Nonlinear Analysis, to appear. 1

[13] L. Rong, LMI approach for global periodicity of neural networks with time-varying delays, IEEE Trans. Circuits Syst. I, 52(2005), 1451-1458. 1

[14] T. Roska, C. Wu, M. Balsi, L. Chua, Stability and dynamics of delay-type general and cellular neural networks, IEEE Trans. Circuits Syst., 39(1992), 487-490. 1

[15] T. Roska, C. Wu, and L. Chua, Stability of Cellular Neural Networks with Dominant Nonlinear and Delay-Type Template, IEEE Trans Circuits Syst. I, 40(1993), 270-272. 1

[16] N. Takahshi, A new sufficient condition for complete stability of cellular neural networks with delay, IEEE Trans. Circuits Syst. I, 47(2000), 793-799.

[17] N. Takahashi and L. Chua, On the complete stability of non-symmetric cellular neural networks, IEEE Trans. Circuits Syst. I, 45(1998), 754-758. 1

[18] Q. Wang and X. Liu, Exponential stability for impulsive delay differential equations by Razumikhin method, J. Math. Anal. Appl., 309(2005), 462-473. 1

[19] Q. Wang and X. Liu, Global Exponential Stability of Impulsive High Order Hopfield Type Neural Networks with Delays, Proceeding of the DSDIC 4th International Conference on Engineering Applications and Computational Algorithms, Watam Press, 2005, 825-830. 1

[20] B. Xu, X. Liu and X. Liao, Global asymptotic stability of high-order Hopfield type neural networks with time delays, Computers and Mathematics with Applications, 45(2003), 17291737. 1

[21] D. Xu and Z. Yang, Impulsive delay differential inequality and stability of neural networks, J. Math. Anal. Appl., 305(2005), 107-120. 1

${ }^{1}$ Department of Computer Science, Mathematics, and Engineering, Shepherd UnIVERSity, SHEPHERDSTOWN, WV 25443,USA

${ }^{2}$ Department of Applied Mathematics, University of Waterloo, Waterloo, OnTARIO N2L 3G1

E-mail address: xzliu@uwaterloo.ca 